\title{
Refractory Priapism Associated with Ingestion of Yohimbe Extract
}

\author{
Amy Myers, $M D^{a}$, Fermin Barrueto, Jr., $M D^{b}$ \\ aDepartment of Emergency Medicine, University of Maryland School of Medicine, Baltimore, MD \\ bClinical Director, Emergency Department, Upper Chesapeake Health System, Bel Air, MD
}

\begin{abstract}
Extracts of the bark of the central African tree Pausinystalia yohimbe contain yohimbine, an indole alkaloid, which is used to treat erectile dysfunction. The reported side effects of over-the-counter preparations of yohimbine include gastrointestinal upset, anxiety, increased blood pressure, headache, agitation, rash, tachycardia, and frequent urination. In this report, we describe a severe case of intractable priapism associated with the ingestion of yohimbe extract. Management required insertion of a proximal cavernosal spongiosum shunt (Quackles shunt) in the operating room.
\end{abstract}

\section{INTRODUCTION}

The incidence of erectile dysfunction is difficult to determine since there are a variety of definitions, but the National Institutes of Health in 2002 has estimated it to be 15-30 million men in the United States. Possible etiologies include increasing age, drugs (citalopram is a common cause) and diseases like diabetes and renal insufficiency. Ease of access combined with the difficulty for the patient to discuss erectile dysfunction with their physicians may lead some to herbal or over-the-counter erectile dysfunction treatments like yohimbine.

Yohimbine is an alkaloid derived from the bark of Pausinystalia yohimbe, a tree indigenous to central Africa. The people of that area have used the bark for centuries as an aphrodisiac and to enhance sexual performance [1,2]. Yohimbe and other natural supplements for erectile dysfunction are attractive alternatives to prescription medications because they are less expensive and can be purchased without a prescription [2]. A meta-analysis published by the American Urological Association in 1998 documented the reported adverse effects of the ingestion of yohimbe [3]. These included gastrointestinal upset (the most common side effect), anxiety, increased blood pressure, headache, agitation, rash, tachycardia, and frequent urination [4]. Anxiety was generally seen in people with a history of anxiety or depression, and increases in blood pressure were generally not experienced by previously normotensive patients [4]. Side effects were reported at the same frequency as among individuals taking placebo (3\%) [4]. In this report, we describe a severe case of intractable priapism following ingestion of a yohimbe product, which was refractory to initial treatments and eventually required definitive surgical intervention.

Keywords: yohimbe, priapism, erectile dysfunction

Notes: There was no outside funding of any kind used for this study.

The authors have no potential financial conflicts of interest to report.

Corresponding Author: Fermin Barrueto, Jr., MD, Department of Emergency Medicine, University of Maryland School of Maryland, 6th Floor, Suite 200, 110 South Paca Street, Baltimore, MD 21201. Email: fbarr001@umaryland.edu 


\section{CASE REPORT}

A 42-year-old man with history of human immunodeficiency virus (HIV) and depression presented to the emergency department (ED) at a Veterans Administration medical center, with an erection that had been sustained for 20 hours. The patient had taken an over-the-counter herbal supplement of yohimbe extract the previous day for sexual enhancement. He had been given prescriptions for highly-active antiretroviral therapy (HAART) for HIV and escitalopram (Lexapro) for depression but was not compliant with those medications. The patient had a history of heroin and cocaine abuse, and stated his last use was several months previously. He denied alcohol use but admitted to daily tobacco use. He said he smoked marijuana that morning, with the intent of easing the pain of his erection, prior to coming to the ED. The patient denied any history of sickle cell disease or recent trauma or infections.

The patient's vital signs on arrival were as follows: temperature, $98.8^{\circ} \mathrm{F}$; pulse, 76 beats/min; mildly elevated blood pressure of 152/87 $\mathrm{mm} \mathrm{Hg}$; respiratory rate, 18 breaths/min. The patient appeared moderately uncomfortable. The remainder of the physical exam was remarkable only for an erect penis and tenderness to palpation of the glans penis. His testicles were not swollen, and his penis was not discolored or cyanotic.

Laboratory investigation revealed a normal complete blood count, electrolytes, and urinalysis. The urine toxicology screen was positive only for tetrahydrocannabinol (THC). It was negative for opiates, cocaine metabolites, amphetamines, barbiturates, benzodiazepines, and methadone.

Intravenous pain medication was given for analgesia. The urology service performed a penile ring block using $25 \mathrm{~mL}$ of $1 \%$ lidocaine without epinephrine. Cavernosal blood gas analysis revealed a $\mathrm{pH}$ of 7.17, pCO2 of 58, and $\mathrm{pO} 2$ of 46 , which are consistent with ischemic priapism (a metabolic acidosis as compared to a normal arterial blood gas). Typically, a cavernosal blood gas will look like a normal arterial blood gas in both nonischemic highflow priapism and a detumesced normal penis. Phenylephrine 400 $\mu \mathrm{g}$ was then injected intracorporeally, and the penile tumescence subsided. The patient's vital signs remained stable throughout the procedure. Within 30 minutes, the erection returned. Three more phenylephrine injections were given intracorporeally, with minimal effect. The corpora was aspirated and flushed with normal saline, and a distal corpus spongiosum shunt (Winter's shunt) was inserted at the bedside. The patient's erection still did not diminish, so he was taken emergently to the operating room for placement of a left proximal cavernosal spongiosum shunt (Quackles shunt). After insertion of the shunt, the priapism resolved immediately. The patient's penis remained flaccid, and he was discharged the following day on amoxicillin/clavulanate (Augmentin) $875 \mathrm{mg}$ by mouth every 12 hours, and acetaminophen/oxycodone (Percocet) $5 / 325$ by mouth every 4 hours, for pain. A follow-up appointment in the urology clinic was scheduled in 2 weeks.

At the 2-week follow-up visit, the patient complained of erectile dysfunction and was started on vardenafil (Levitra) $2.5 \mathrm{mg}$ prior to sexual activity. At the 6-month follow-up visit, the patient stated that the 2.5-mg dose of vardenafil had been only minimally effective. The urologist increased the vardenafil dosage to $5 \mathrm{mg}$ prior to sexual activity, with follow-up in the primary care clinic for titration of the medication.

\section{DISCUSSION}

This patient's depression and use of citalopram were risk factors that caused or exacerbated his erectile dysfunction. His priapism temporally coincided with the use of this herbal preparation and he did not have any of the diseases that are typically associated with priapism, e.g., sickle cell disease or leukemia. He was also not actively using any drugs typically associated with priapism, such as trazadone, androgens, cocaine, ethanol, antihypertensives and antipsychotics. He did test positive for marijuana, but how this contributed to his priapism cannot be determined. Yohimbine's complex mechanism of action can explain the resultant refractory priapism in this patient.

The human corpus cavernosum has functional alpha-2 adrenergic receptors. Antagonist actions on these receptors are responsible for cavernosal smooth muscle contraction and detumescence $[2,5,6]$. Yohimbine is an alpha-2 adrenoreceptor antagonist that works primarily at the presynaptic site, which is thought to enhance penile erection. When given intracavernosally, yohimbe has no effect on penile erection [4]. This is an interesting aside, as multiple intracorporeal injections of phenylephrine, an alpha-agonist, was not effective for this patient. Its mechanism of action appears to be related not only to its alpha-2 adrenergic blockade but also to its central cholinergic activity $[2,5,6]$ Peripherally, the alpha-2-adrenergic blockade may block norepinephrine-induced contraction of the corporeal cavernosal smooth muscle [2]. There also appears to be a direct effect on the relaxation of smooth muscle by the increase in release of nitrous oxide $[3,7]$.

Yohimbine's mechanism of action has been studied for many years, but the drug has not undergone rigorous human clinical trials [2]. In the human studies that have been performed, the sample size has generally been regarded as too low to allow any conclusion as to yohimbe's effectiveness. It has been postulated that the reason for the lack of more thorough study is that yohimbine cannot be patented and therefore economic incentives are lacking [2,3].

When yohimbine is taken orally, peak levels are reached in 10 to 15 minutes [1]. The half-life is estimated between 20 and 35 minutes $[2,7]$. This quick onset of action, along with easy accessibility and low cost, make over-the-counter yohimbe supplements more appealing than prescription erectile dysfunction medications $[2,3]$.

\section{CONCLUSION}

Given yohimbe's alpha-2-adrenoreceptor antagonism, intractable priapism could be a likely side effect of the ingestion of products containing this compound. 


\section{REFERENCES}

1. Guay AT, Spark RF, Jacobson J, et al. Yohimbe treatment of organic erectile dysfunction in a dose-escalation trial. Int J Impot Res. 2002;14:25-31.

2. Morales A. Yohimbe in erectile dysfunction: the facts. Int J Impot Res. 2000;12(suppl 1):S70-S74.

3. McKay D. Nutrients and botanicals for erectile dysfunction: examining the evidence. Altern Med Rev. 2004;9:4-16.
4. Ernst E, Pittler MH. Yohimbe for erectile dysfunction: a systematic review and meta-analysis of randomized clinical trials. J Urol. 1998;159:433-436.

5. Hatzichristou DG, Pescatori ES. Current treatments and emerging therapeutic approaches in male erectile dysfunction. BJU Int. 2001;88(suppl 3):11-17.

6. Adeniyi AA, Brindley GS, Pryor JP, et al. Yohimbe in the treatment of orgasmic dysfunction. Asian J Androl. 2007;9:403-407.

7. Tam SW, Worcel M, Michael W. Yohimbine: a clinical review. Pharmacol Ther. 2001;91:215-243. 